\title{
Segmental Bronchial Diameter Measurement at Inspiration-Expiration Phase by Low-dose MSCT in Healthy Volunteers
}

\author{
Li He, Qingyun Ren, Zhai Liu* \\ Department of Radiology, The First Hospital of Hebei Medical University, Shijiazhuang, China \\ Email address: \\ heli801104@163.com (Li He), ren_qingyun@163.com (Qingyun Ren), tuliphappy@yeah.net (Zhai Liu) \\ *Corresponding author
}

To cite this article:

Li He, Qingyun Ren, Zhai Liu. Segmental Bronchial Diameter Measurement at Inspiration-Expiration Phase by Low-dose MSCT in Healthy Volunteers. International Journal of Biomedical Materials Research. Vol. 7, No. 2, 2019, pp. 72-77. doi: 10.11648/j.ijbmr.20190702.12

Received: February 13, 2019; Accepted: April 23, 2019; Published: August 30, 2019

\begin{abstract}
Objective: This study aimed to evaluate the normal range of quantitative CT in measuring the airway diameter at inspiration-expiration phase using 3D imaging in healthy adults. Materials and Methods: 68 healthy volunteers, including 42 males and 26 females, with the mean age of 49.3 (SD14.7) years, had undergone low-dose CT scan at full inspiration and the end of expiration. All CT scans were performed within the range from the lung apices to the diaphragm, at a tube potential of $120 \mathrm{kVp}$ and an effective mAs of 60. Afterwards, all CT images were analyzed using the 3D software (FUJIFILM Corporation SYNAPSE), and the airway tree was generated according to the automated region-growing technique, which was perpendicular to the cross section measurement of the segmental bronchus in bilateral lower lobes. Meanwhile, the imaging of segmental bronchus was classified as at inspiration and expiration phase in male group, and at inspiration and expiration phase in female group. Moreover, the Din-L and Din-S of the segmental bronchus were analyzed quantitatively. Difference in the segmental bronchial diameter between the inspiration and expiration phase group with the same sex was evaluated with paired-t test, while that between the male and female groups was analyzed by a 2-tailed unpaired $t$ test. In addition, the impact of age, height, weight and BMI on Din-L and Din-S was assessed through multiple linear regression analysis. Results: 378 segmental branches in the lower lobe of lung from male group and 234 from female group were shown using the low-dose multi-slice spiral CT (MSCT) scan. All segmental bronchi were detected automatically using the 3D software. Our results suggested that, Din-L and Din-S in segmental bronchus of both male and female groups at inspiration phase were larger than those at expiration phase; and those in male group were greater than those in female group at both inspiration and expiration phases $(\mathrm{P}<0.01)$. Meanwhile, the average of Din-L and Din-S was markedly related to age at both inspiration and expiration phases. Conclusions: The normal reference values and the likely ranges are determined for segmental bronchus diameter in the lower lobe of adult lung at inspiration-expiration phase. The average of Din-L and Din-S is evidently correlated with age and sex at both inspiration and expiration phases. The segmental bronchus diameter at inspiration phase in adolescents is larger than that at expiration phase.
\end{abstract}

Keywords: Computer Tomography, Bronchus, Diameter, Low-Dose

\section{Introduction}

With the development of medical imaging technology, multi-slice spiral CT (MSCT) has been extensively applied in clinic, which allows to accurately diagnose numerous diseases, especially for lung and bronchial diseases. Chronic obstructive pulmonary disease (COPD), asthma, tracheobronchial amyloidosis, as well as other bronchial diseases, can result in bronchial wall thickening and lumen narrowing, eventually leading to ventilation dysfunction.

Based on the understanding of lung disease and the development of bronchoscope, bronchial diameter has attracted increasing attention. Currently, the size and lumen area of bronchus can be measured in vivo through CT images. In previous studies, bronchial dimensions are acquired through manual measurements, rather than by using semiautomatic segmentation software, which can reduce operator dependency and produce more objective 
measurements $[1,2]$. MSCT has higher temporal and spatial resolution, and completely identical spatial resolution of the image can be attained in all directions. As a result, chest MSCT images can be reconstructed at any direction, so as to observe the bronchial lumen. Currently, bronchial measurements can be obtained from the MSCT scan of lungs using the post-processing software [3, 4]. In some studies, CT is employed for bronchial measurements in healthy volunteers as well as patients with bronchial asthma and COPD [3-8]. However, many existing studies have focused on the wall thickness, others have measured the lumens of central bronchi, segmental bronchi, or the small airway at inspiration phase. Moreover, some studies showed the changes in lung volume and bronchial diameter in COPD were quantitative measured at inspiration and expiration phase [9-11], while some studies have used low-dose CT scans [12-13]. Specifically, Wall thickness and airway intraluminal area of six segmental bronchi (B1, B2, B3, B8, B9, and B10) in the right lung of in patients with COPD at inspiration phase were measured automatically using three-dimensional computed tomography [14]. Nonetheless, the segmental bronchial diameter has not been reported within the normal range of expiratory and inspiratory phase scanning.

Consequently, this study aimed to observe and measure the bronchial lumen displayed by low-dose CT combined with the 3D post-processing technology, which could provide the normal reference range of the segmental bronchial diameter in bilateral lower lobes of lung at inspiration and expiration phases; besides, this study also aimed to observe the relationships of bronchial diameter with respiratory status, gender, age, weight and height.

\section{Materials and Methods}

\subsection{Subjects}

A total of 68 healthy adults undergoing low-dose chest CT, including 42 males and 26 females, with the age ranging from 24 to 83 years, (mean, $49.3 \pm 14.7$ years), were collected from the Physical Examination Center at the First Hospital of Hebei Medical University from September to December 2017. Among them, those with normal findings on chest CT scans and with no known history of lung disease or surgery were enrolled into this study. Additionally, subjects with thoracic neoplasm, COPD, or postoperative chest diseases, and those with diseases affecting bronchial diameter were excluded from this study.

\subsection{Image Acquisition}

All subjects received volumetric CT at full inspiration and at the end of normal expiration in supine position using the 64 multi-detector scanners (Light speed, GE Healthcare Corporation), with no contrast in the craniocaudal direction. All CT scans were carried out at a tube potential of $120 \mathrm{kV}$ and an effective mAs of 60 at inspiration and expiration phases. In addition, the reconstructed slice thickness and slice intervals were $0.625 \mathrm{~mm}$, respectively, while the matrix was $512 \times 512$, the field of view (FOV) was $50 \mathrm{~cm} \times 50 \mathrm{~cm}$, and standard algorithm reconstruction was applied. The scan quality was assessed for the presence/absence of motion artifacts, adequacy of inspiration/expiration, inclusion of the entire lung, and adherence to the scanning protocol.

\subsection{Image Analysis}

All CT reconstructed images were analyzed using the SYNAPSE 3D software (FUJIFILM Corporation), which could automatically remove the mediastinum, major blood vessels, trachea, as well as the thoracic wall tissues. Besides, the airway tree could also be extracted and displayed automatically. The bronchi in the bilateral lower lobes of lungs were selected, respectively, and the plane perpendicular to the bronchial axis was selected for measurement. Typically, the software could also automatically recognize and generate the bronchial luminal long (Din-L) and short diameter (Din-S), and the airway diameter measurements across the middle third of each segment were averaged. Every scan was visually assessed for the accuracy of segmental bronchi, the integrity of airway tree, and accuracy of airway labels. The automated measurements were also manually edited if necessary. In our study, the airway tree extracted by the software could well display the sub-segmental bronchi at inspiration phase, partial sub-segmental bronchi at expiration phase could not be accurately assessed by the software. Then, the segmental bronchial diameter was selected to analyze at inspiration and expiration phases.

Moreover, the luminal long and short diameters of the right lower lobe dorsal segmental bronchi (RB6), anterior basal segmental bronchi (RB7), internal basal segmental bronchi (RB8), posterior basal segmental bronchi (RB9), external basal segmental bronchi (RB10) and left lower lobe dorsal segmental bronchi (LB6), anterior internal basal segmental bronchi (LB7+8), external basal segmental bronchi (LB9), and posterior basal segmental bronchi (LB10) were measured in both male and female subjects at expiration and inspiration phases.

\subsection{Statistical Analysis}

The Statistical Package for the Social Sciences (version 19.0, SPSS Inc, Chicago, IL, USA) was employed for all statistical analyses. A difference of $P<0.05$ was considered as statistically significant. Data were expressed as mean \pm standard deviation $(\overline{\mathrm{x}} \pm \mathrm{S})$. Differences in the segmental bronchial diameter between the inspiration and expiration phase groups with the same sex were evaluated using paired- $t$ test, while those between the male and female groups were analyzed with the 2-tailed unpaired t test. In addition, the effects of age, height, weight and body mass index (BMI) on the average of Din-L and Din-S were assessed through multiple linear regression analysis.

\section{Results}

All images obtained from 68 subjects undergoing low-dose 
MSCT were processed using the FUJIFILM Corporation SYNAPSE 3D software, so as to extract the bronchial tree. A total of 378 and 234 segmental branches in the lower lobe of lung in male and female groups were detected, respectively, using low-dose MSCT scan and 3D reconstruction software. Din-L and Din-S of the segmental bronchi in bilateral lower lobes of male subjects were larger than those of female subjects at both inspiration and expiration phases $(\mathrm{P}<0.05)$.
Din-L and Din-S of the segmental bronchi in bilateral lower lobes in male and female subjects at inspiration phase were greater than those at expiration phase $(P<0.05$, Table 1$)$.

Upon multiple regression (Table 2), the average of Din-L and Din-S was markedly related to age at inspiration and expiration phases $(P=0.012,0.031)$, but that was not markedly correlated with height $(\mathrm{P}=0.096,0.107)$, weight $(\mathrm{P}=0.245$, $0.185)$ and $\mathrm{BMI}(P=0.431,0.164)$.

Table 1. Comparison of diameter in bilateral lower segmental bronchi at inspiration-expiration phase between male and female subjects.

\begin{tabular}{|c|c|c|c|c|c|c|c|c|c|}
\hline \multirow{2}{*}{ Sex } & \multirow{2}{*}{$\mathbf{N}$} & \multicolumn{2}{|l|}{ Din-L } & \multirow{2}{*}{$\mathbf{t}$} & \multirow{2}{*}{$P$} & \multicolumn{2}{|l|}{ Din-S (mm) } & \multirow{2}{*}{$\mathbf{t}$} & \multirow{2}{*}{$\boldsymbol{P}$} \\
\hline & & inspiration & expiration & & & inspiration & expiration & & \\
\hline $\mathrm{M}$ & 378 & $6.1 \pm 1.9$ & $5.4 \pm 1.7$ & 12.062 & 0.000 & $4.9 \pm 1.4$ & $4.3 \pm 1.3$ & 11.683 & 0.000 \\
\hline $\mathrm{F}$ & 234 & $5.2 \pm 1.7$ & $4.7 \pm 1.6$ & 5.888 & 0.000 & $4.1 \pm 1.2$ & $3.8 \pm 1.2$ & 5.171 & 0.000 \\
\hline $\mathrm{t}$ & & 6.275 & 4.678 & & & 6.971 & 4.822 & & \\
\hline $\mathrm{P}$ & & 0.000 & 0.000 & & & 0.000 & 0.000 & & \\
\hline
\end{tabular}

Din- $\mathrm{L}=$ bronchial luminal long diameter, Din-S= bronchial luminal short diameter.

Table 2. Multiple Regression Analysis for average of Din-L and Din-S at inspiration-expiration phase.

\begin{tabular}{llllllll}
\hline \multirow{2}{*}{ Model } & Inspiration & & & & \multicolumn{2}{l}{ Expiration } \\
\cline { 2 - 7 } & B & std.error & t & P & B & std.error & t \\
\hline Age & 0.016 & 0.006 & 2.603 & 0.012 & 0.012 & 0.006 & 2.201 \\
Height & 0.152 & 0.090 & 1.689 & 0.096 & 0.138 & 0.084 & 1.636 \\
Weight & -0.135 & 0.115 & -1.173 & 0.245 & -1.144 & 0.108 & -1.339 \\
BMI & 0.388 & 0.328 & 1.182 & 0.242 & 0.431 & 0.306 & 1.408 \\
\hline
\end{tabular}

Din-L= bronchial luminal long diameter, Din-S= bronchial luminal short diameter.

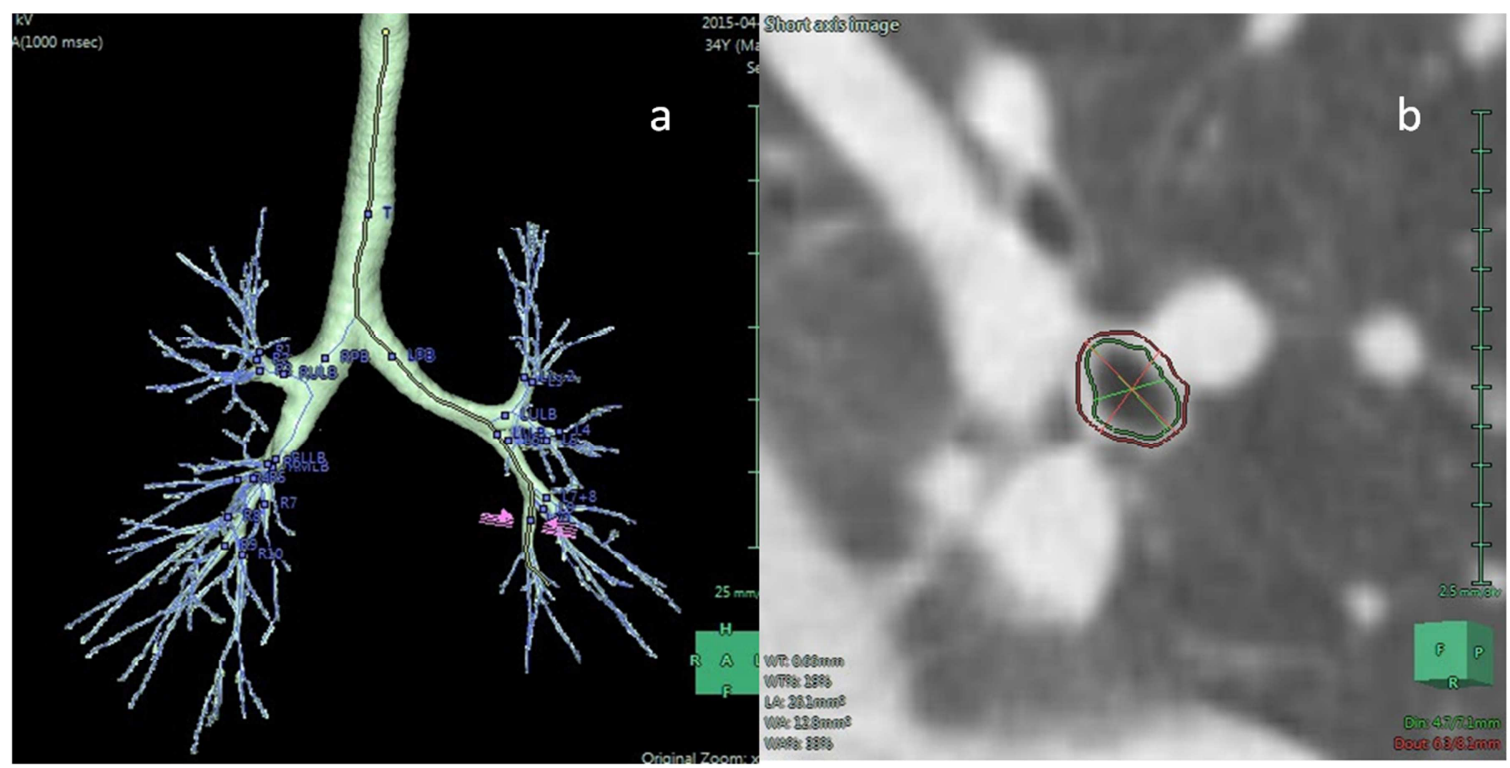

Figure 1. a. bronchus tree image of 3-dimensional reconstructions b. measurement of the airway diameter at LB10.

\section{Discussion}

The trachea and bronchi are constituted by the gradually thinning branches, while the airway can be divided into about 23 generation, and the whole trachea and bronchial system is like an inverted tree, which is often called the airway tree. Some scholars have investigated the bronchi using autopsy specimens, bronchography, bronchoscope, traditional sectional CT, as well as the currently available MSCT [15-18]. Applying MSCT can achieve a wide range of isotropic imaging within a short period of time, which can also reconstruct images at any angle to observe the tissue structure of trachea and bronchi. Therefore, the application of MSCT allows for investigating the anatomy based on the large samples in vivo. In recent years, MSCT and post-processing technology have been widely applied in trachea and bronchi research [3, 11, 14, 19].

CT examination for central lung cancer, endobronchial tuberculosis and other diseases can display the narrow segmental bronchial lumen, while that on COPD and asthma can show the small airway stenosis, bronchial wall thickening and stenosis [18]. Tracheobronchitis can result in strictures in 
the trachea and bronchi, whereas allergic bronchopulmonary aspergillosis may induce central bronchiectasis. In this study, the internal diameter of segmental bronchus was quantitatively measured by $\mathrm{CT}$, which could provide a normal reference value for diagnosing bronchial diseases.

All images from the 68 subjects undergoing low-dose MSCT were processed using the FUJIFILM Corporation SYNAPSE 3D software, so as to extract the bronchial tree. The biphasic bronchi to the bronchial lumen of bilateral lower lobes of lungs were clearly displayed, among which, 378 branches of bilateral lower lobe bronchi were displayed in male and 234 in females. Our results indicated that the inner bronchial diameters in male at inspiration and expiration phases were larger than those in women, and the differences between the two phases were statistically significant, which might be ascribed to different body size and breathing pattern of men from those of women. In addition, our study also suggested that the long and short diameters of the segmental bronchi in both males and females at inspiration phase were markedly greater than those at expiration phase, which could be attributed to the increased pulmonary pressure during expiration, bronchial muscle contraction and luminal reduction, nonetheless, the bronchus could still maintain the oval shape due to the support of the bronchial cartilage. Zou LG et al. [20] showed that high resolution CT could clearly display the morphological structure of the segmental and sub-segmental bronchi, but only the luminal diameter of end-inspiratory bronchi was measured, while the changes at expiration phase were not observed. Most patients can breathe freely at the time of bronchoscope, but the insertion depth of bronchoscope into the bronchi was affected by the smaller bronchial lumen at expiration. Therefore, compared with the inspiration state, it is more important to measure the diameter of normal bronchi at expiration state. Liu M et al. [21] had studied the airway diameter by $\mathrm{CT}$ in children aged $<5$ years, and their results revealed that age and body weight were the important factors affecting airway diameter in children, but there was no difference in the airway diameter of children between different sexes and different respiratory phase, which were inconsistent with our results. It was speculated that the reason might be related to the immature lung and respiratory muscles, as well as the small lung capacity in children. In our study, the segmental airway diameter was slightly increased with age, which was in agreement with that by Zach [3] showed that the wall area percent (WA\%) of segmental bronchi was slightly decreased with the increase in age. We speculated that the muscle atrophy in bronchi and the decrease in bronchial elasticity with age might be responsible for the increased airway diameter with age.

In recent years, some studies have been carried out to examine the pulmonary volume and small airway in patients with COPD and asthma by $\mathrm{CT}$ at inspiration and expiration phases $[11,22,23]$. Therefore, the use of CT for quantification of airway analysis in patients with COPD and healthy individuals is expected to increase in future. However, the increase in radiation exposure of the population due to this increased CT usage has attracted wide attention. What we need to do is to reduce radiation dose while maintaining adequate image quality. Notably, the low dose CT protocol at $50 \mathrm{mAs}$ can produce consistent screening results with those of the standard dose CT protocol (150 mAs), which can there by support the routine use of low dose chest CT protocol [24]. Xie X et al. [18] had used low-dose CT (60 mAs) to measure the small airway wall thickness in patients with chronic bronchial disease, they found that male heavy smokers with chronic respiratory symptoms in lung cancer screening, who are ate high-risk of chronic bronchitis, have bronchial wall thickening in airways with a luminal diameter of $5-10 \mathrm{~mm}$ but not in larger airways. Chen $\mathrm{H}$ et al. [25] used the low dose (40 $\mathrm{mAs}$ ) lung $\mathrm{CT}$ to investigate the correlation of bronchial lumen with the pulmonary function parameters in COPD, they found that some HRCT parameters are correlated to some PFT parameters. In this study, low-dose CT scan at a tube current of $60 \mathrm{mAs}$ was performed, and the CT image could clearly show the wall thickness and airway intra luminal area of airway, in the meantime, the radiation dose to the subjects could be markedly reduced. Trachea and segmental bronchi are composed of cartilages, muscles, connective tissues and mucosa; on CT scans, airways appear as the dark tubular structures surrounded by the bright wall, which are distinct from the inflated lung tissue and air in the cavity. Therefore, low-dose CT can clearly display the structures of trachea and bronchial lumen. Our findings showed that, the bronchial trees above the sub-segmental bronchi in all subjects could be displayed and measured completely by CT at inhalation, which was consistent with the results from Zou LG. In expiratory CT, only the bronchial tree above the segment can be displayed and measured completely, while some sub-segmental bronchi can not be displayed clearly, which can be ascribed to the smaller bronchial lumen below the segment, as well as the decreased difference in density between the bronchial lumen and the surrounding tissues.

Most segmental bronchi are not completely perpendicular to the transverse axial plane, the oblique section of the bronchus will be displayed on the transverse axial CT image. As a result, the true lumen diameter can not be clearly displayed on axial CT image. In this study, the airway trees could be extracted through using the $3 \mathrm{D}$ software by one-click, and the orthogonal section image perpendicular to the segmental bronchi was selected from the 3D bronchial tree image. In addition, the long and short diameters of the airway in the selected layers were measured by the software automatically, and the accuracy of the inner bronchial diameter measured by the 3D image was much higher than that by the $2 \mathrm{D}$ image [26].

Nonetheless, several limitations should be noted in this study. Firstly, the final sample size was smaller for female than for male, which might lead to a somewhat unbalanced comparison between different sexes. Secondly, only the lower lobe bronchial lumen diameters in bilateral lungs were measured, while bronchial diameters in other lobes were not measured. Thirdly, although our dataset was diverse, information about smoking was not recorded, while smoking might be a confounding factor of diameter in segmental 
bronchi. Moreover, we did not consider the patients' physical size during CT scans performed using fixed mAs (60 mAs), which may have produced different noise levels during segmental bronchi measurements. Besides, our results were applicable only to adults.

In conclusion, this study shows that CT examination on the segmental bronchus can be performed at inspiration and expiration phases using the low dose CT scans at $60 \mathrm{mAs}$. The segmental bronchial diameter can be measured accurately using the $3 \mathrm{D}$ post-processing software. The normal reference values and the likely ranges are determined for segmental bronchus diameter in the lower lobe of adult lung at inspiration-expiration phase. The average of Din-L and Din-S is evidently correlated with age and sex at both inspiration and expiration phases. Part of sub-segmental bronchi and small airways on expiratory chest $\mathrm{CT}$ are poorly displayed; therefore, we recommend that chest $\mathrm{CT}$ at inspiration phase can be used in measurement of sub-segmental bronchi and small airways, so as to diagnose diseases involving the small airways.

$\mathrm{He} \mathrm{Li}$ contributed to experimental design and implementation, data collection and collation, and manuscript drafting. Liu Zhai was responsible for experimental implementation, evaluation, and data collection. and Ren Qingyun contributed to quality control and calibration. There was no conflict of interest in this article.

\section{References}

[1] Naidich DP, Zinn WL, Ettenger NA, et al. Basilar segmental bronchi: thin-section CT evaluation [J]. Radiology, 1988, 169 (1): 11-16.

[2] Matsuoka Y, Ookubo T, Ookubo K, et al Thin-section computed tomography of the bronchi: right middle lobe, left lingular division, and lower lobe [J]. Rinsho Hoshasen 1989, 34 (7): 799-803.

[3] Zach J A, Jd Newell J, Schroeder J, et al. Quantitative CT of the Lungs and Airways in Healthy Non-smoking Adults [J]. Investigative Radiology, 2012, 47 (10): 596-602.

[4] Hackx M, FRANCOTTE, Dorothée, Severo Garcia T, et al. Effect of Total Lung Capacity, Gender and Height on CT airways measurements [J]. The British Journal of Radiology, 2017, 90 (1076): 20160898.

[5] Nambu A, Zach J, Schroeder J, et al. Quantitative computed tomography measurements to evaluate airway disease in chronic obstructive pulmonary disease: Relationship to physiological measurements, clinical index and visual assessment of airway disease [J]. European Journal of Radiology, 2016, 85 (11): 2144-2151.

[6] Philip K, Oliver W, Wielpütz Mark O, et al. Quantitative CT detects changes in airway dimensions and air-trapping after bronchial thermoplasty for severe asthma [J]. European Journal of Radiology, 2018, 107 (8): 33-38.

[7] Kuo W, Ciet P, Andrinopoulou E R, et al. Reference Values for Central Airway Dimensions on CT Images of Children and Adolescents [J]. AJR Am J Roentgenol, 2018, 210 (2): 423-430.
[8] Mi W, Zhang C, Wang H, et al. Measurement and Analysis of the Tracheobronchial Tree in Chinese Population Using Computed Tomography [J]. PLOS ONE, 2015, 10 (6): e0130239.

[9] Matsuoka S. Airway dimensions at inspiratory and expiratory multisection CT in chronic obstructive pulmonary disease: correlation with airflow limitation [J]. Radiology, 2008, 248 (3): 1042-1049.

[10] Kloth C, Thaiss W M, Ditt H, et al. Segmental bronchi collapsibility: computed tomography-based quantification in patients with chronic obstructive pulmonary disease and correlation with emphysema phenotype, corresponding lung volume changes and clinical parameters [J]. Journal of Thoracic Disease, 2016, 8 (12): 3521-3529.

[11] Chae EJ, Kim TB, Cho YS, et al. Airway Measurement for Airway Remodeling Defined by Post-Bronchodilator FEV1/FVC in Asthma: Investigation Using Inspiration-Expiration Computed Tomography [J]. Allergy Asthma Immunol Res, 2011, 3 (2): 111-117.

[12] Xie X, Dijkstra AE, V onk JM, et al. Chronic respiratory symptoms associated with airway wall thickening measured by thin-slice low-dose CT [J]. AJR. 2014, 203 (4): W383-390.

[13] Song S K, Jin G Y, Li Y Z, et al. CT Quantification of Lungs and Airways in Normal Korean Subjects: [J]. Korean Journal of Radiology, 2017, 18 (4): 739-748.

[14] Karayama M, Inui N, Mori K, et al. Respiratory impedance is correlated with morphological changes in the lungs on three-dimensional CT in patients with COPD [J]. Scientific Reports, 2017, 7: 41709.

[15] Scannel JG, Boston MD, Mass. A study of variations of the bronchopulmonary segments in the left upper lobe [J]. J Thorac Surg, 1947, 31: 530-537.

[16] Boyden EA, Hamre CJ, Minn M. An analysis of variations in the bronchovascular patterns of the middle lobe in fifty dissected and twenty injcted lungs [J]. J Thorac Surg, 1951, 21 (2): $172-188$.

[17] Boyen EA. The nomenclature of the bronchopulmonary segments and their blood supply [J]. Dis Chest, 1961, 39 (1): $1-6$.

[18] Xie X, Dijkstra AE, Vonk JM, et al. Chronic respiratory symptoms associated with airway wall thickening measured by thin-slice low-dose CT [J]. AJR. 2014, 203 (4): W383-390.

[19] Bauer C, Eberlein M, Beichel R R. Graph-Based Airway Tree Reconstruction From Chest CT Scans: Evaluation of Different Features on Five Cohorts [J]. IEEE Trans Med Imaging, 2015, 34 (5): 1063-1076.

[20] Zou LG, Zhang XS, Yang H, et al. Quantitative CT study of segmental and subsegmental bronchi in normal subjects [J]. ChongQing Medicine, 2012, 4 (11): 8-10 (in Chinese).

[21] Liu M, Yan WC, Yang Y, et al. Measurement of airway dimensions in children under $5 \mathrm{y}$ of age: a CT study with controlled ventitation [J]. Radiologic Practice, 2015, 30 (3) 275-278 (in Chinese).

[22] CP Hersh, GR Washko, RS Estépar, et al. Paired inspiratory-expiratory chest CT scans to assess for small airways disease in COPD [J]. Respiratory Research, 2013, 14 (1): 42 . 
[23] Kirby M, Yin Y, Tschirren J, et al. A Novel Method of Estimating Small Airway Disease Using Inspiratory-to-Expiratory Computed Tomography [J]. Respiration, 2017, 94 (4): 336-345.

[24] Kubo T, Ohno Y, Nishino M, et al. Low dose chest CT protocol $(50 \mathrm{mAs})$ as a routine protocol for comprehensive assessment of intrathoracic abnormality. [J]. European Journal of Radiology Open, 2016, 3 (C): 86-94.
[25] Chen H, Chen G Q, Zeng Q S, et al. Quantitative Assessment of Airway Pathology in Subjects With COPD Using Low-Dose High-Resolution Computed Tomography. [J]. Respiratory Care, 2017, 62 (7): 953-962.

[26] Lee JW, Son JS, Choi JW, et al. The comparison of the longs and diameters of main bronchi measured from two-dimensional and three-dimensional images in the same patients [J]. Korean J Anesthsiol. 2014, 66 (3): 189-194. 